\title{
Bone Metabolic Biomarkers-Based Diagnosis of Osteoporosis Caused by Diabetes Mellitus using Support Vector Machine
}

\section{Chuan Wang}

Naval Medical Center of PLA, Shanghai, China

\section{Taomin Zhang}

State Key Laboratory of Bioelectronics, Jiangsu Laboratory for Biomaterials and Devices, School of Biological Science and Medical Engineering, Southeast University, Nanjing, China

\section{Xuan Liu}

School of Medicine,SoutheastUniversity,Nanjing 210009, China

\section{Lei Miao}

School of Computer Science and Engineering, Southeast University, Nanjing, China

\section{Deyu Zhou}

School of Computer Science and Engineering, Southeast University, Nanjing, China

\section{Peng Wang}

Department of Sports Medicine and Adult Reconstructive Surgery, Drum Tower Hospital affiliated to Medical School of Nanjing University, Nanjing, China

\section{Yibo Zhang}

Department of Sports Medicine and Adult Reconstructive Surgery, Drum Tower Hospital affiliated to Medical School of Nanjing University, Nanjing, China

\section{Qing Jiang}

Department of Sports Medicine and Adult Reconstructive Surgery, Drum Tower Hospital affiliated to Medical School of Nanjing University, Nanjing, China

\section{Yezi Hu}

Department of endocrine secretion, Zhongda Hospital affiliated to Southeast University, Nanjing, China

\section{Han Yin}

Department of endocrine secretion, Zhongda Hospital affiliated to Southeast University, Nanjing, China hui Jin

Zhongda Hospital affiliated to Southeast University

Jianfei Sun ( $\nabla$ sunzaghi@seu.edu.cn )

Southeast university https://orcid.org/0000-0003-2204-0479

\section{Research}


Keywords: Bone Turnover Markers, Support Vector Machine, Type 2 Diabetes, Osteoporosis

Posted Date: March 24th, 2020

DOI: https://doi.org/10.21203/rs.3.rs-17981/v1

License: (c) (1) This work is licensed under a Creative Commons Attribution 4.0 International License. Read Full License 


\section{Bone Metabolic Biomarkers-Based Diagnosis of Osteoporosis Caused by Diabetes Mellitus using Support Vector Machine}

Chuan Wang1\#, Taomin Zhang2\#, Xuan Liu3, Lei Miao4, Deyu Zhou4, Peng Wang5, Yibo Zhang5, Qing Jiang5, Yezi Hu6, Han Yin6, Hui Jin6*, Jianfei Sun2*

ıNaval Medical Center of PLA, Shanghai, China

2State Key Laboratory of Bioelectronics, Jiangsu Laboratory for Biomaterials and Devices, School of Biological Science and Medical Engineering, Southeast University, Nanjing, China

3SchoolofMedicine, SoutheastUniversity, Nanjing210009, China

4School of Computer Science and Engineering, Southeast University, Nanjing, China

5Department of Sports Medicine and Adult Reconstructive Surgery, Drum Tower Hospital affiliated to Medical School of Nanjing University, Nanjing, China

6Department of endocrine secretion, Zhongda Hospital affiliated to Southeast University, Nanjing, China

\section{*Correspondence to}

Jianfei Sun, PhD, State Key Laboratory of Bioelectronics Jiangsu Laboratory for Biomaterials and Devices Department of Biological Science and Medical Engineering, Southeast University, Nanjing, China. E-mail: sunzaghi@seu.edu.cn.

Hui Jin, MD, Department of endocrine secretion, Zhongda Hospital affiliated to Southeast University, Nanjing, China. E-mail: jinhuison@126.com. 


\begin{abstract}
Background: Diabetes has significant effects on bone metabolism. Both type 1 and type 2 diabetes can cause osteoporotic fracture. However, it remains challenging to diagnose osteoporosis in type 2 diabetes by bone mineral density which lacks regular changes. Seen another way, osteoporosis can be ascribed to the imbalance of bone metabolism, which is closely related to diabetes as well. Method: Here, to assist clinicians in diagnosing osteoporosis in type 2 diabetes, an efficient and simple SVM model was established based on different combinations of biochemical indices, including bone turnover makers, calcium and phosphorus, etc. The classification performance was measured using several evaluations. Results: The predicting accuracy rate of final model is above $88 \%$, with feature combination of Sex, Age, BMI, TP1NP and OSTEOC. Conclusion: Experimental results show that the model has come to an anticipant result for early detection and daily monitoring on type 2 diabetic osteoporosis.
\end{abstract}

Keyword: Bone Turnover Markers, Support Vector Machine, Type 2 Diabetes, Osteoporosis.

Running title: AI (artificial intelligence) for diseases diagnosis 


\section{Background}

Diabetes and osteoporosis are two of the most common diseases in modern age. Moreover, the osteoporosis is often complication of diabetes.[1] Traditionally, dual-energy x-ray absorptiometry (DEXA) test is the gold standard to evaluate and diagnose osteoporosis by checking bone mineral density (BMD). However, it has been found that the BMD-test is not always amenable to diagnose the osteoporosis caused by diabetes. Type 1 diabetes mellitus (T1DM) inhibits the formation of new bone so that the BMD is decreased, which resembles the common osteoporosis [2]. In contrast with T1DM, the BMD variety of type 2 diabetes mellitus (T2DM) is often irregularly [3]. The BMD seems sometime normal or higher [4]. However, for T2DM patients, the risk of osteoporotic fracture is higher than that expected in clinic and the therapeutic plan is different $[5$, 6]. Thus, accurate evaluation of bone health by BMD in the T2DM patients remains a critical link in clinic. An effective diagnostic method of osteoporosis that can be used for the T2DM patients routinely is required.

The serological test is common in the routine physical examination. Because both the diabetes and the osteoporosis are metabolic diseases, the serological test should be sensitive to body changes. Moreover, studies revealed that the glucose level is closely associated with the bone health as well [7-9]. Diabetes could result in vitamin-D deficiency [10], and hyperglycemia could suppress osteoblastic formation [11]. Thus, the test of bone turn-over markers (BTMs) is possible to indicate the osteoporosis from the T2DM patients. Nowadays, the test of BTMs has not been widely clinically used in contrast with the DEXA [12] resulting from lack of the specific biomarkers for the osteoporosis [13]. To solve this issue, an alternative is to use computer to 
analyze big data so that the accuracy of diagnostic result can be enhanced. With development of artificial intelligence, this strategy has been applied in diverse areas of healthcare [14-16].

Here, we utilized support vector machine (SVM) to analyze the database of T2DM patients and the algorithm can effectively predict the osteoporosis from the T2DM patients. SVM, also called as large margin classifier, basically is to minimize the distance between classification hyperplane and the support vectors, which are the closest points to the hyperplane. With robust classification ability and excellent generalization performance, SVM only needs to set a few parameters to tune the model based on hundreds of samples [17]. Here, we proposed a SVM-based method to diagnose the osteoporosis from T2DM based on the BTMs of serological testing. Different combinations were generated as inputs sets according to the importance of testing items (Introduction to the common BTMs was shown in Supporting Information, Note S1). Multiple SVM models with different input sets were established, among which the combination of TP1NP (total procollagen I n-terminal propeptide), OSTEOC (osteocalcin), gender, age and BMI (body mass index) showed the best performance. The diagnosis accuracy can reach $88 \%$. Surprisingly, ALP (alkaline phosphatase) that is the common biomarker for osteogenesis was found to have insignificant effect on the classification model. These results demonstrated that computer science will boost the traditional means of diagnosis and play an increasingly important role in the diagnosis of chronic diseases.

\section{Methods}

\section{Datasets}


Data used in this study was collected from the Department of Endocrine, Zhongda Hospital affiliated to Southeast University. The dataset distribution was shown in Figure 1. In modeling dataset, 202 qualified samples were collected from patients during Jan. 2016 to Mar. 2018, and each sample consists of 10 attributes including gender, age, BMI, levels of Ca, P, ALP, TP1NP, PICP, OSTEOC and VIT-D.

\section{Implementation design}

After the modeling dataset was established, SVM algorithm was used for classification task. The flowchart of data processing was shown in Figure 2. The classification was done based on Scikit learning which is a software package of machine learning in Python. The detailed steps were given as follows.

\section{1) Data Preprocessing}

Every samples which diagnosis was T2DM complicated by osteoporosis was labeled as positive one (1), falling into positive class. If the diagnosis was just T2DM, the sample was labeled as negative one (-1), falling into negative class. If the gender was female, the sample was labeled as 1. Otherwise, it was 0 . Because age has significant influence on risk of osteoporosis both for men and women, the age was grouped and weight of each group was set, as shown in Supporting Information, Table S1. The setting of weight was dependent upon the sample number each group. For different range of attributes, the data were normalized in order to avoid the influence of large numeric attributes on the calculation results, which was as Eq. (1)

$$
y^{\prime}=\text { lower }+(\text { upper }- \text { lower }) \times \frac{y-\min }{\max -\min }
$$


This formula converted the eigenvalue to a specific interval, where y is the data before scaling, and $\mathrm{y}^{\prime}$ is the scaled data. lower and upper are the lower bound and upper bound of the given interval, respectively. In this study, the importance of all attributes is considered as the same at first. The data were scaled into $[0,1]$.

\section{2) Modification of imbalanced data}

The practical data are always imbalanced, especially data collected directly from the clinic. There is always a tendency for the classifiers to get biased in order to achieve higher prediction accuracy. There were 40 samples or $19.8 \%$ of positive class and 162 samples or $80.2 \%$ of negative class in our dataset. Synthetic Minority Oversampling Technique (SMOTE) was adopted due to the limited sample size in this experiment [18]. After SMOTE, the sample size of minority class was increased from 40 to 162 . Finally, the dataset contained 324 samples in total.

\section{3) Selecting of important features}

The impact of each feature on classification result is different. Therefore, based on the original data, importance of the features was judged using tree-based estimators. The features were ranked in the order of importance as shown in Figure 3. The weight was larger if the feature was more important. To ensure the classification accuracy and reduce the cost of computing, data dimensions were reduced by ignoring less important features. Six combinations of the attributes were tested which were called as Test 1-6, as shown in Supporting Information, Table S2. Here, 323 samples were used as training set and 1 sample was for testing. After repeating 202 times in each test, classification performance was finally obtained. 


\section{4) Parameter optimization}

To map the original low-dimensional space into the high-dimensional feature space, the training set was modeled by various kernel functions including radial basis function, polynomial, and sigmoid. In order to improve the generalization ability, soft margin was introduced by adjusting the penalty coefficient C. Parameter C represents the relative importance of classification risk and error rate, which means $\mathrm{C}$ is the trade-off between the maximum margin and the noise tolerance. The larger $\mathrm{C}$ means the classification is more rigorous, yielding less mistakes. Secondly, when the gaussian kernel function was selected, the complexity of the model can be adjusted by changing the parameter gamma. The larger value of gamma means the original data are mapped into the higher dimensions and the boundary of classification is more complex. To obtain the best parameters of each model, cross validation was used. As mentioned above, 323 samples in training set were divided into 5 sub-sample sets. One sub-sample set was selected randomly as the verification one while the other sets were used for training. After multiple training and verification, average training score was obtained. The model with the highest training score was considered as the best one. By completing the above operations, the SVM model was established.

\section{5) Evaluation of the classification performance}

The classification performance was evaluated by using four metrics: accuracy, precision, recall and area under the receiver operating characteristic curve (ROC-AUC value) based on the confusion matrix. The accuracy, the precision and the recall were calculated by Eq. (2)-(4):

$$
\text { accuracy }=\frac{T P+T N}{T P+F N+T N+F P}
$$


precision $=\frac{T P}{T P+F P}$

recall $=\frac{T P}{T P+F N}$

where $T P$ refers to True Positive (number of positive labeled samples that were predicted correctly), $T N$ refers to True Negative (number of negative labeled samples that were predicted correctly), FP refers to False Positive (number of negative labeled samples that were predicted wrongly) and $F N$ refers to False Negative (number of positive labeled samples that were predicted wrongly). The accuracy score means the percentage of correct classification. One drawback of the accuracy is lack of the potential distribution of testing values. To get a comprehensive understanding of classification performance, the precision and the recall score are used to see whether the classifier identified the positive case or negative case correctly. The precision refers to the percentage of real positive samples in samples marked as true positive. The recall refers to the percentage of true positive samples in the real positive data set. The ROC-AUC value is a score obtained from the receiver operating characteristic (ROC) analysis. ROC plots the function of true positive rate (TPR) and false positive rate (FPR) when verifying threshold. The increase of TPR comes at the cost of increasing FPR. The area under the ROC curve (ROC-AUC) can be used as another evaluation of the model accuracy. Values of ROC-AUC always lies between 0 and 1 among which the values above 0.9 indicate excellent prediction, between 0.7 and 0.9 good, between 0.5 and 0.7 poor and any value below 0.5 is considered no better than a random guess [19].

\section{Results and Discussion}




\section{Importance of Features}

Based on Figure 3, the top 5 attributes were TP1NP, age, P content, gender and OSTEOC, which are of great guiding significance in diagnosis of T2DM complicated with osteoporosis. Interestingly, the VIT-D, the BMI and especially the ALP were found insignificantly important. Moreover, both TP1NP and PICP were reported to indicate bone formation [20]. However, the AI results showed that TP1NP is more sensitive than PICP in BMT-based diagnosis of T2DM complicated with osteoporosis. Surprisingly, ALP, as the commonly preferred biomarker of osteogenesis, was at the bottom. Also, BMI seems less closely associated with osteoporosis rather than that people always thought [21]. Besides, Ca, PICP and VIT-D also showed less importance than expected in this issue. These results will be helping for physicians in clinical diagnosis of T2DM complicated osteoporosis.

\section{Classification results}

The SVM-based classification algorithms are often evaluated using confusion metrics as shown in Supporting Information, Fig. S1. For evaluation and comprehensive analysis of each classifier, the classification performances of 6 tests were listed in Supporting Information, Table 3 and plotted in Figure 4. It was seen that Test 1, 2 and 3 possessed over $85 \%$ accuracy and over $50 \%$ precision. There was a positive correlation between the number of attributes and classification accuracy. The accuracy score of Test 1 , which included 10 attributes, was improved remarkably than Test 4, 5 and 6, which included 4 or 5 attributes. At the same time, it should be noted that the precision in all tests was relatively low because of the imbalanced data in verification. As the number of features decreasing, the really positive samples were more difficult to be distinguished 
from the positively marked samples.

Here, one valuable conclusion is that the not all the testing items are needed. Compared with

Test 1, Test 2 with 7 attributes showed the nearly same classification accuracy and ROC-AUC value. The recall of Test 2 was even higher that of Test 1, indicating that it is feasible to use a few most influential testing items for diagnosis. With the same number of dimension, Test 3 obtained higher score on classification accuracy and ROC-AUC value than Test 4 and 5, which demonstrated that TP1NP from Test 3 is better as an attribute than PICP and ALP from Test 4 and 5. This may suggest that TP1NP is more specific as an evaluating indicator for bone metabolism in BTMs testing.

In addition, too few attributes are inadequate to yield correct results with the SVM algorithm. Test 6, which included the important attributes, showed bad classification performance. Under the premise without decrease of testing performance, the reduction of items is good. Actually, resulting from complex interactions among the organs and systems, biochemical information from clinical tests may be redundant. By AI technology, some diseases can be diagnosed by relatively simpler testing items but significantly reduce the cost. Furthermore, AI can establish connections between the phenotype of serological testing and the development of disease. This is important for the diagnosis of degenerative diseases, such as osteoporosis, because there remains no highly specific biomarker for these diseases.

\section{Conclusion}

In this paper, SVM algorithm was tried to classify the osteoporosis from the T2DM relying on several serological items and personal information. The accuracy can be over $85 \%$, showing 
promising potentials for the diagnosis of T2DM complicated with osteoporosis in clinic. This method is cheap, safe and extendible. Interestingly, some cases different from common sense were found, such as ALP playing an insignificant role in the AI-based diagnosis. These results will be helpful for the clinical and POCT diagnosis of osteoporosis, deepening the investigation of pathological mechanism.

\section{Abbreviations}

DEXA: dual-energy x-ray absorptiometry; BMD: bone mineral density; BTMs: bone turnover markers; SVM: support vector machine; ALP: alkaline phosphatase; TP1NP: total procollagen I n-terminal propeptide; PICP: carboxyterrninal propeptide of I pmcollagen; VIT-D: vitamin-D.

\section{Authors' contribution}

JS and HJ proposed the idea. CW and TZ did the experiments. XL, YZ and QJ helped the understanding about T2DM complicated with osteoporosis. LM and DZ helped to make the program. YH and HY provided the clinical samples. TZ wrote the manuscript under the help of JS. CW and PW helped to modify the manuscript.

\section{Author details}

1Naval Medical Center of PLA, Shanghai, China; 2State Key Laboratory of Bioelectronics, Jiangsu Laboratory for Biomaterials and Devices, School of Biological Science and Medical Engineering, Southeast University, Nanjing, China; 3SchoolofMedicine, SoutheastUniversity, Nanjing210009, China 4School of Computer Science and Engineering, Southeast University, Nanjing, China; 5Department of Sports Medicine and Adult Reconstructive Surgery, Drum Tower Hospital affiliated to Medical School of Nanjing University, Nanjing, China; 6Department of endocrine secretion, Zhongda Hospital affiliated to Southeast University, Nanjing, China. 


\section{Acknowledgements}

Not applicable.

\section{Competing interests}

All the authors declare that there is no conflict of interest related to the work presented in this article.

\section{Availability of data and materials}

All data generated or analyzed during this study are included in this published article.

\section{Consent for publication}

Not applicable.

\section{Ethics approval and consent to participate}

All methods in this study were carried out in accordance with relevant guidelines and regulations.

\section{Funding}

Not applicable.

\section{Reference}

1. Chen Z, Zhao GH, Zhang YK, Shen GS, Xu YJ, Xu NW: Research on the correlation of diabetes mellitus complicated with osteoporosis with lipid metabolism, adipokines and inflammatory factors and its regression analysis. European Review for Medical and Pharmacological Sciences 2017, 21: 3900-3905.

2. Miazgowski T, Pynka S, Noworyta-ZietaraL: Bone mineral density and hip structural analysis in type 1 diabetic men. European Journal of Endocrinology 2007, 156(1): 123-127.

3. Rakic V, Davis WA, Chubb SAP: Bone mineral density and its determinants in diabetes: the Fremantle Diabetes Study. Diabetologia 2006, 49(5): 863-871.

4. Yamamoto M: Insights into bone fragility in diabetes: The crucial role of bone quality on skeletal strength. Endocrine Journal 2015, 62(4): 299-308. 
5. Schwartz AV, Sellmeyer DE, Ensrud KE: Older Women with Diabetes Have an Increased Risk of Fracture: A Prospective Study. The Journal of Clinical Endocrinology \& Metabolism 2001, 86(1):32-38.

6. Yokomoto-Umakoshi M, Kanazawa I, Kondo S: Association between the risk of falls and osteoporotic fractures in patients with type 2 diabetes mellitus. Endocrine Journal 2017, 64(7): 727-734.

7. Kruse C: The New Possibilities from "Big Data" to Overlooked Associations Between Diabetes, Biochemical Parameters, Glucose Control, and Osteoporosis. Current Osteoporosis Reports 2018, 16: 320-324.

8. Liu MY, Lu YH, Cheng XL, Ma LC, Miao XY, Li N, Sun BR, Yan ST, Li J, Li CL: Relationship between abnormal glucose metabolism and osteoporosis in Han Chinese men over the age of 50 years. Clinical Interventions in Aging 2019, 14: 445-451.

9. van Bommel EJM, de Jongh RT, Brands M, Heijboer AC, den Heijer M, Serlie MJ, van Raalte DH: The osteoblast: Linking glucocorticoid-induced osteoporosis and hyperglycaemia? A post-hoc analysis of a randomised clinical trial. Bone 2018, 112: 173-176.

10. Eliades M, Pittas AG: Vitamin D and Type 2 Diabetes. Journal of Steroid Biochemistry \& Molecular Biology 2017, 173: 280-285.

11. Jackuliak P, Payer J: Osteoporosis, Fractures, and Diabetes. International Journal of Endocrinology 2014, 2014: 1-10.

12. Kanis JA, Burlet N, Cooper C: European guidance for the diagnosis and management of osteoporosis in postmenopausal women. Osteoporosis International 2008, 19(4): 399-428.

13. Kuo TR, Chen CH: Bone biomarker for the clinical assessment of osteoporosis: recent developments and future perspectives. Biomarker Research 2017, 5(1): 2-9.

14. Lewandowski J, Arochena HE, Naguib RNG: Logic-Centered Architecture for Ubiquitous Health Monitoring. IEEE Journal of Biomedical and Health Informatics 2014, 18(5): 1525-1532.

15. Li QG, Zhao KY, Bustamante CD, Xin Ma X: Xrare: a machine learning method jointly modeling phenotypes and genetic evidence for rare disease diagnosis. Genetics in Medicine 2019, 21: 2126-2134.

16. Safdar S, Zafar S, Zafar N, Khan NF: Machine learning based decision support systems (DSS) for heart disease diagnosis: a review. Artificial Intelligence Review 2018, 50: 597-623.

17. Noble WS: What is a support vector machine?. Nature Biotechnology 2006, 24: 1565-1567.

18. Verbiest N, Ramentol E, Cornelis C, Herrera F: Preprocessing noisy imbalanced datasets using SMOTE enhanced with fuzzy rough prototype selection. Applied Soft Computing 2014, 22: 511-517.

19. Zhu W, Lomsadze A, Borodovsky M: Ab initio gene identification in metagenomic sequences. Nucleic Acids Research 2020, 38(12): e132-e144.

20. Price CP, Thompson PW: The role of biochemical tests in the screening and monitoring of osteoporosis. Annals of Clinical Biochemistry 1995, 32: 244-260.

21. Frederiksen AG, Lysgaard AL, Iversen E, Jørgensen NR, Schwarz P: BMI might be overestimated in elderly and old osteoporosis patients suffering vertebral fracture(s). European Geriatric Medicine 2013, 4: S127-128. 


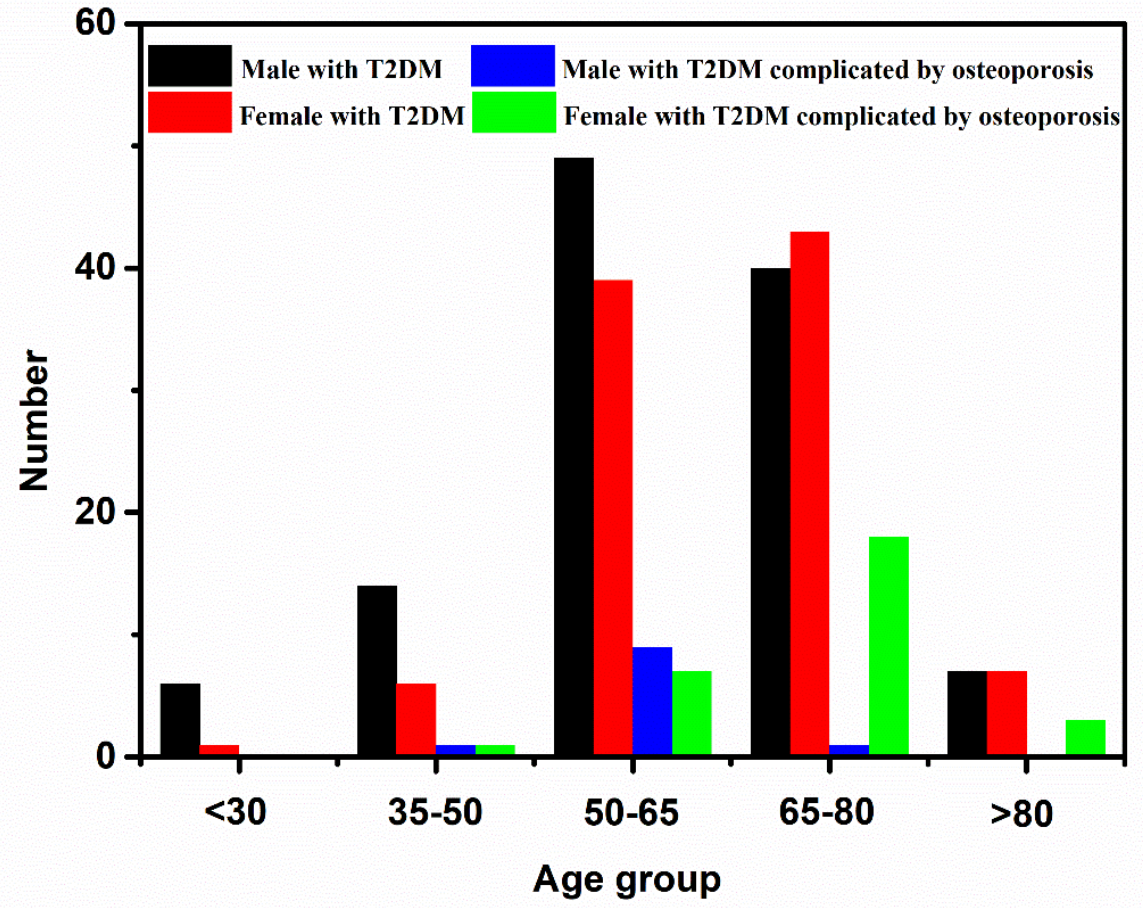

Figure 1 Sample distribution in the dataset 


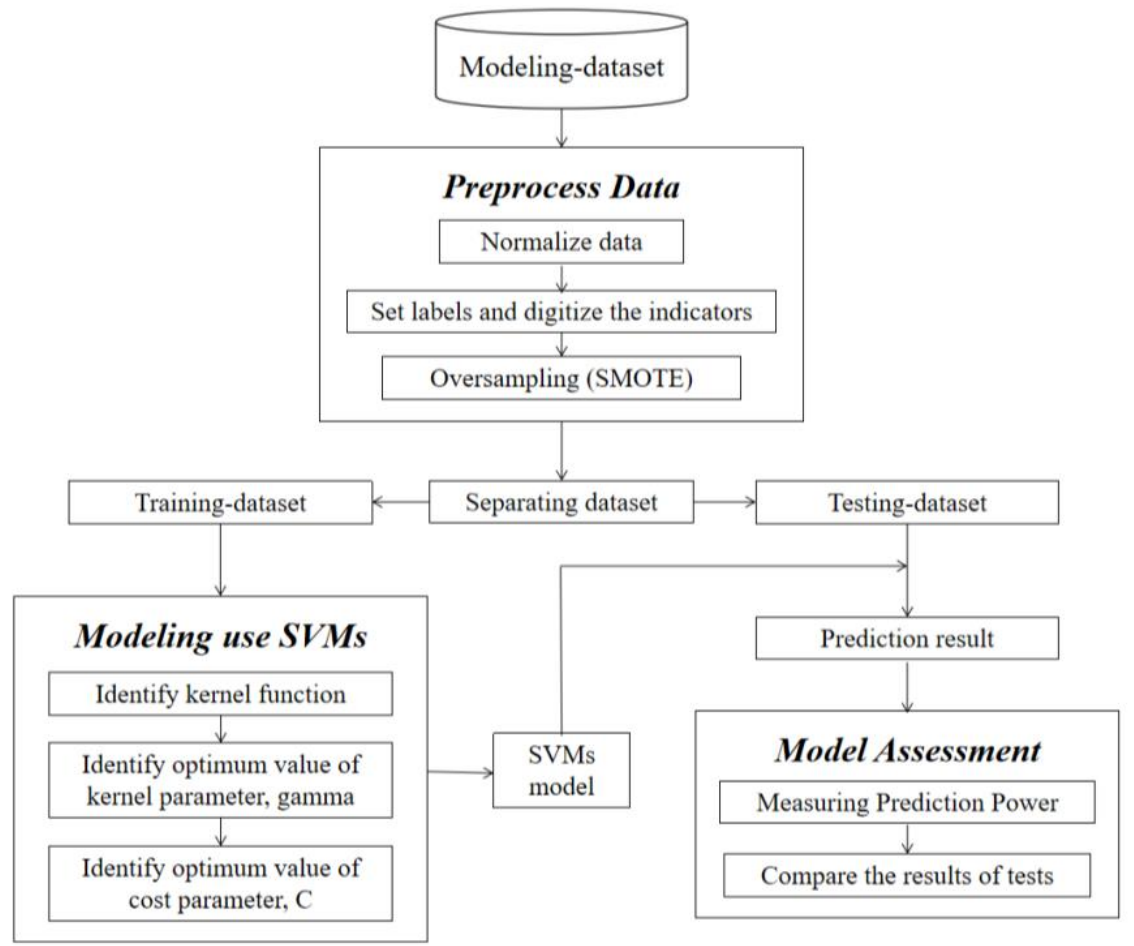

Figure 2 Flowchart of data processing for osteoporosis classification with SVM

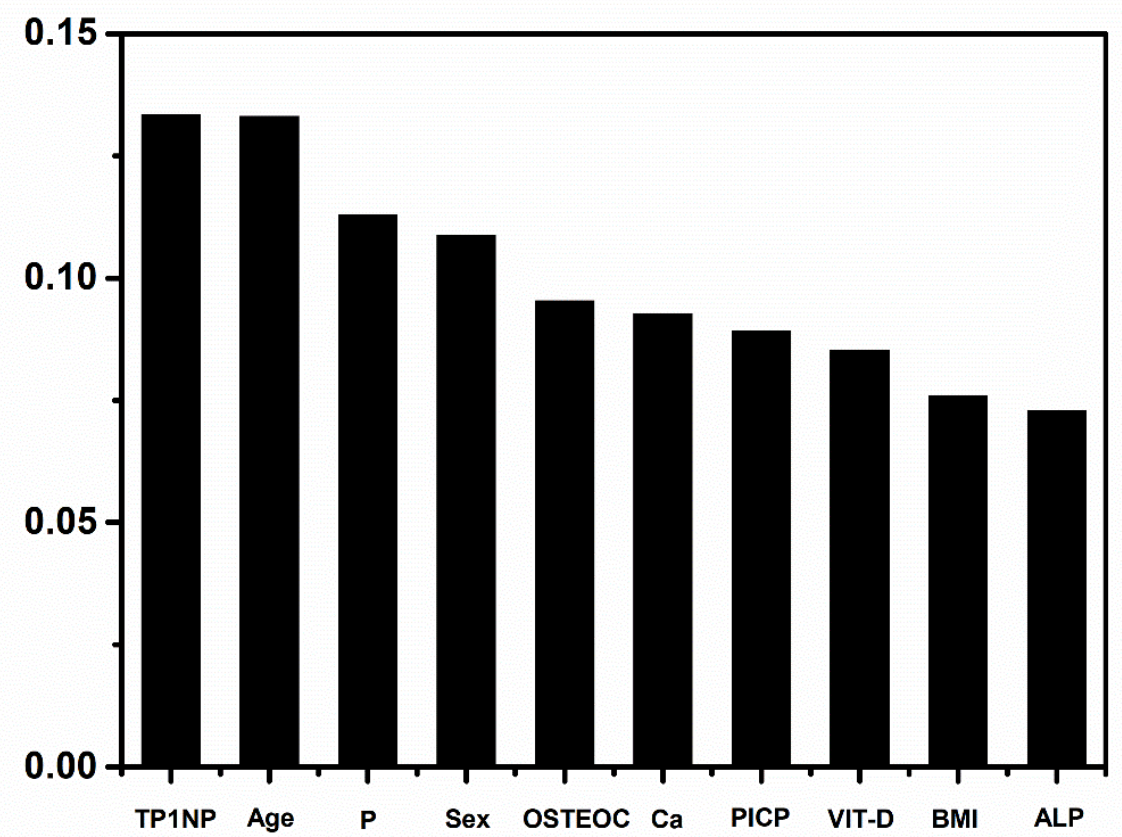

Figure 3 Ordering of importance for the testing items 


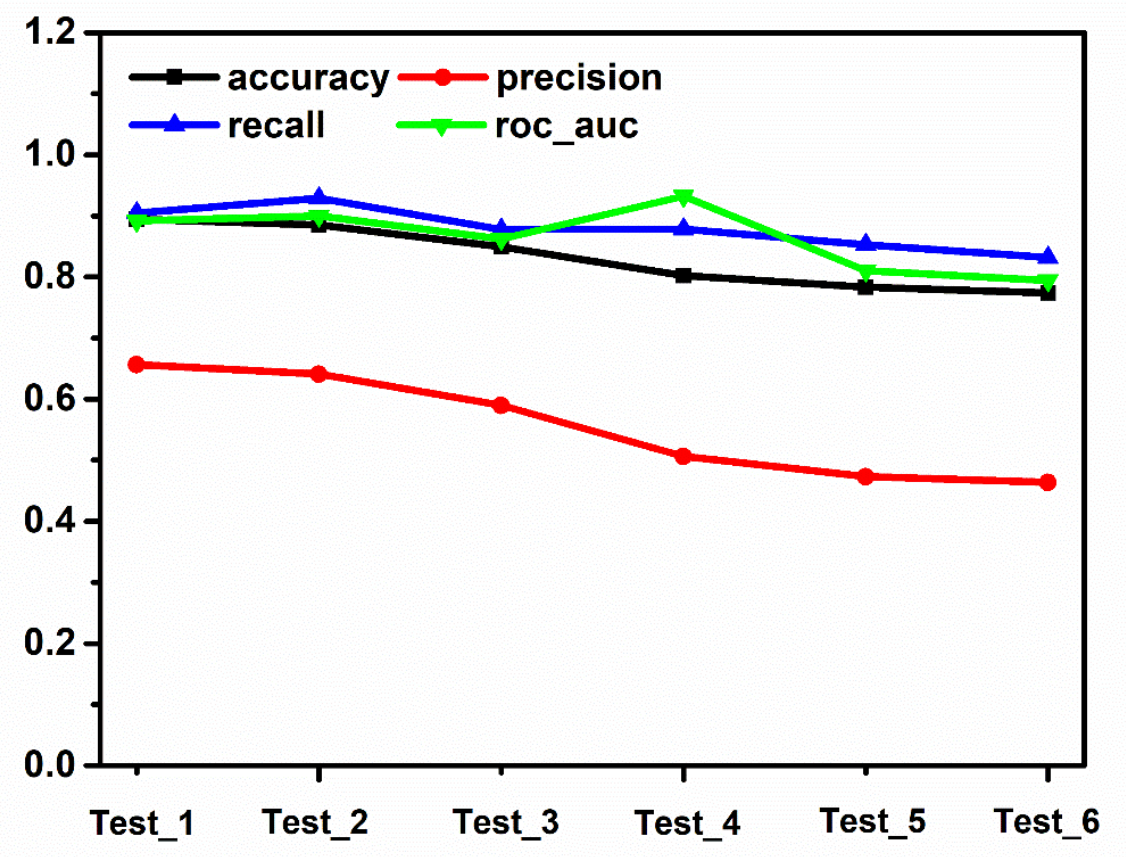

Figure 4 Classification performances of the six combinations with different attributes 
Figures

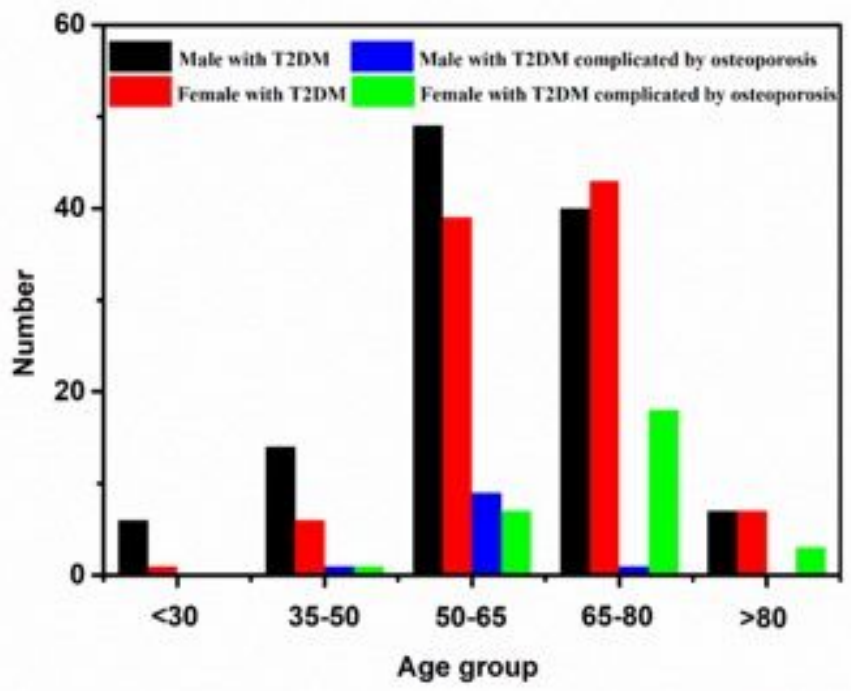

\section{Figure 1}

Sample distribution in the dataset

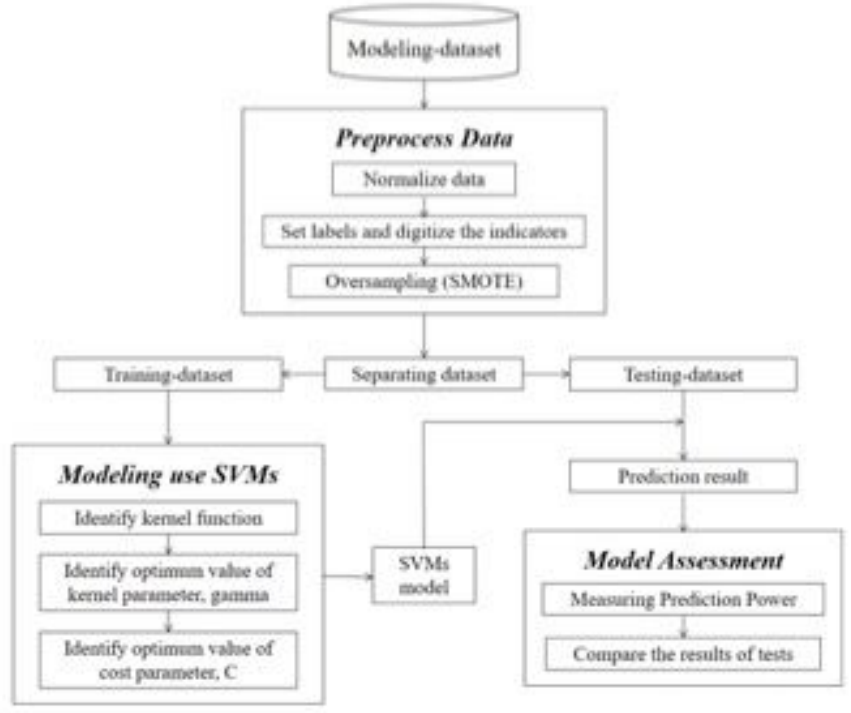

Figure 2

Flowchart of data processing for osteoporosis classification with SVM 


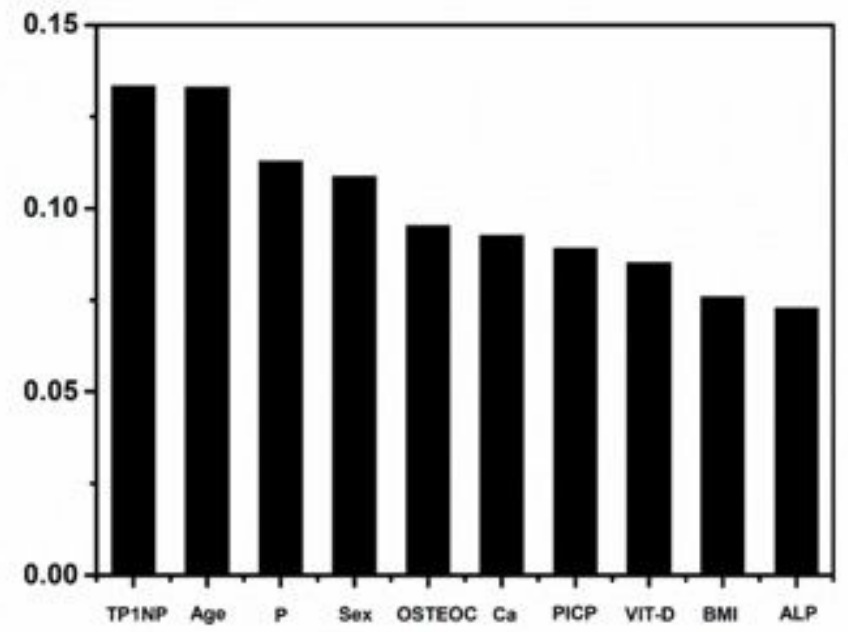

Figure 3

Ordering of importance for the testing items

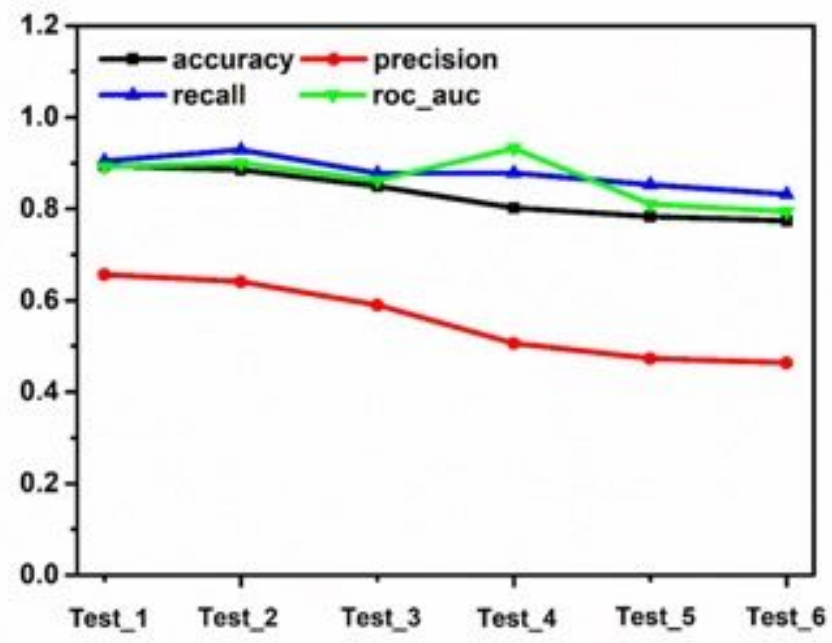

Figure 4

Classification performances of the six combinations with different attributes

\section{Supplementary Files}

This is a list of supplementary files associated with this preprint. Click to download.

- SupportingInformation.docx 